\title{
Antifungal effect of Satureja khuzestanica Jamzad essential oil on oral candidiasis in immunosuppressed rats
}

\author{
TEIMUR NAJIBZADEH* ${ }^{*}$ MOHAMMAD HOSSEIN YADEGARY
}

Department of Mycology

Faculty of Medicine

Tarbiat Modares University

School of Medical Sciences

Tehran, Iran

"corresponding author: +989163609807, e-mail: teimurnajibzadeh@gmail.com

\section{Summary}

Introduction: The antimicrobial, antibacterial, antioxidant, antihyperlipidaemic, antidiabetic, anti-inflammatory and analgesic effects of Satureja khuzestanica (Lamiaceae) have been investigated in numerous studies.

Objective: The antifungal effects of S. khuzestanica essential oil (SKJO) in immunosuppressed rats suffering from oral candidiasis were investigated.

Methods: A fungal suspension with a density of $3 \times 10^{8} \mathrm{CFU} / \mathrm{ml}$ was taken from the isolate of a case with acute vaginitis. In order to achieve a medicinal formulation, accurate twice the minimum inhibitory concentration (MIC) of SKJO and 10 times MIC of nystatin was used with $0.8 \%$ agar as a base material. In this study, 35 rats divided into 5 groups each of 7 were used. The assessment of level of infection and specification of the effects of treatment were performed using microbiological and histopathological methods as well as clinical examinations.

Results: Our results show that carvacrol is the main compound of SKJ essential oil. Lesions completely disappeared in both treatment groups (SKJO and nystatin) after 8 days of treatment. Furthermore, in groups treated with SKJO and nystatin, the quantities of mean logarithm of colony forming unit (CFU) were $85.62 \%$ and $90.62 \%$, respectively, in comparison with placebo group. Also, histopathological properties suggested no infection in groups who had clean and normal dorsal tongue mucosa.

Conclusions: Using SKJO with twice accuracy it was concluded that MIC in immunosuppressed rats was quite successful in a time of a week and equal with that of a nystatin treatment with an accuracy of 10 times the MIC.

Key words: Satureja khuzestanica Jamzad, essential oil, nystatin, placebo, candidiasis, MIC

Słowa kluczowe: Satureja khuzestanica Jamzad, nystatyna, placebo, grzybica, MIC 


\section{INTRODUCTION}

Candidiasis is a range of opportunistic fungal diseases that occur in susceptible cases, ranging in form from simple infections to systemic ones [1]. These infection appear as acute, sub-acute or chronic in skin, nails, oral and vaginal mucosa, bronchi, lungs and digestive system, infecting other major organs such as kidneys, liver and hearth in its systemic form [2]. Nowadays, candidiasis is one of leading infectious diseases in the world and accounts for 25 to $50 \%$ of nosocomial infections in ICU and 8 to $15 \%$ of overall nosocomial infections [3].

Mucosal candida infections increased considerably with the prevalence of Acquired Immunodeficiency Syndrome (AIDS) and more than $90 \%$ of HIV cases suffering from oral-pharyngeal candidiasis [4]. Also, these infections are more prevalent in cases resulting from underlying factors such as leukemia, diabetes mellitus, prolonged antibiotic and corticosteroid treatments, AIDS, pregnancy, burns and transplants. These infections range from mucosal colonization to aggressive and fatal infections. Of the different clinical forms of candida infections, cutaneous and mucosal candidiasis are the most prevalent ones. In mucosal forms, vaginal candidiasis and oral thrush in cutaneous form, nail infection (Onychomycosis) are more prevalent than others [5]. C. albicans is the most significant type separated from oral lesions and the leading cause of oral mucosal infection $[6,7]$. Nowadays, drugs used in modern treatment of candidiasis belong to polyens and azoles $[8,9]$. The polyens group, including amphotericin and nystatin, exhibits toxic renal and hepatic complications. The azoles group, including itraconazole and fluconazole, are less toxic, yet exhibit lower treatment success due to the resistance of different candida groups (especially C. glabrata, C. tropicalis) [10, 11]. For these reasons, experts are looking for potent drugs with no side-effects to treat this disease and this has led to a special attention to herbs and drugs derived from them. Herbs have been used for a long time to cure many diseases due to a variety of useful compounds in them. One such useful compound in herbs is essential oil or volatile oils with a host of biological effects. Antimicrobial and antifungal effects of essential oils have been reported repeatedly and are attributed to different compounds such as cineole, camphor, linalool, $\alpha$-pinene, $\beta$-pinene, borneol, limonene, carvacrol, thymol, camphene, $\alpha$-terpineol, etc. [12]. Satureja khuzestanica Jamzad (SKJ) - Lamiaceae - was identified for the first time in 1985 in the south of Lorestan Province (southwestern Iran) and named by Jamzad (1994). The antimicrobial, antibacterial, antioxidant, antihyperlipidemic, antidiabetic, anti-inflammatory and analgesic effects of S. khuzestanica have been investigated in numerous studies $[13,14]$. The main goal of this study was to evaluate the antifungal effects of SKJO (Satureja khuzestanica Jamzad essential oil) on inhibiting candida growth both in in vitro (laboratory cultures) and in vivo (in immunosuppressed rats infected with oral candidiasis).

\section{MATERIAL AND METHODS}

\section{Plant material, essential oil extraction and main component of SKJO by GC and GC-MS}

The aerial parts of SKJ were collected during spring season at the flowering stage of the plant from Pol E Dokhtar (Lorestan province, Iran) and dried in shade at laboratory temperature.

For the extraction of the essential oil, dried aerial parts (100 g) of plant were subjected to hydro-distillation method by Clevenger type apparatus for $5 \mathrm{~h}$. SKJO is colorless or yellowish liquid with a specific weight of 0.895 to 0.913 . It is soluble in dimethyl sulfoxide (DMSO) [15]. In order to identify the compounds, the extracted essential oil was injected into GC-Mass apparatus. The findings will be discussed in the results section. Nystatin was used as a positive control in the treatment of oral candidiasis of rats. Pure nystatin powder was obtained from Iran Darou pharmaceuticals, manufactured in Italy.

\section{Antifungal evaluation of SKJO and measure- ment of MIC and MFC}

In order to prepare the culture environment to determine the minimum inhibitory concentration (MIC) for SKJO and for nystatin, $30 \mathrm{~g}$ of Sabouraud Dextrose Agar Broth Base (Merck, Germany) was solved in 1 liter of distilled water. Then it was autoclaved at $121^{\circ} \mathrm{C}$ and the pressure of $15 \mathrm{psi}$ for $15 \mathrm{~min}$. In order to determine the MIC value, in this study the Broth Macrodilution method was used. Since SKJO is slightly water-soluble, DMSO was used as a solvent. DMSO must have $6 \%$ density for it not to have antifungal effects, since, according to control experiments on DMSO solvent in this study, it was determined that DMSO does not exhibit 
antifungal effects in densities lower than $6 \%$. In order to determine the minimum fungicidal concentration (MFC), super optimised broth (SOB) culture environment was used.

Stages of the determination of the density of SKJO included: a) providing young and active micro-organisms, b) preparing a standard microbial suspension for insemination including a tube with $2 \mathrm{ml}$ Sabouraud Dextrose Broth (SDB) culture environment with $2000 \mathrm{CFU} / \mathrm{ml}$ of active C. albicans cells [16], c) dilution and insemination of micro-organisms including SKJO and nystatin dilution as well as positive control and control solvent all of which had $40 \mu \mathrm{l}$ of standard and active microbial suspension. After this stage, all tubes were homogenized using Vortex apparatus and then were put inside an incubator for $48 \mathrm{~h}$ at $30^{\circ} \mathrm{C}$. After this period, the MIC results were determined visually. The lowest density of the materials, more than those in which no candida growth was visible, was determined as the MIC [16]. Also, MFC of SKJO procedure for approving MIC results included stages as follows: after visual measurement of the MIC, $100 \mu \mathrm{l}$ were taken from tubes with MIC density or higher and distributed by loop under laminar air flow in plates with Sabourauds Dextrose Agar (SDA) culture. Next, thy were eventually inserted into plates in the incubator at $30^{\circ} \mathrm{C}$ for $48 \mathrm{~h}$. The lowest density with no apparent colonial growth was determined as MFC of C. albicans. Also MIC and MFC values for nystatin (positive control) were determined [17]. In order to boost the accuracy in all MIC and MFC tests for SKJO and nystatin, they were run three times to observe similar results.

In order to achieve a medicinal formulation for SKJO, final density twice the MIC of SKJO and for nystatin, 10 times the MIC of nystatin was used. The base material for this formulation was $0.8 \%$ agar (1 to 9). These medical formulations were used to treat oral candidiasis twice a day each time $0.5 \mathrm{ml}$ for a whole week. It is noteworthy that in order to achieve better treatment results, the drug was made on a daily basis and used freshly [18]. For the formulation of placebo, a combination of sterile physiological serum and melted agar with a ratio of 1 to 9 was used.

Preparation of immunosuppressed rats and experimental set-up in Italy.

\section{Antifungal evaluation of SKJO and measure- ment of MIC and MFC}

Since C. albicans is an opportunistic fungal organism and causes sickness in rats with a weak immune system, immunosuppressed rats were used in this study (of Wistar strain, all male, eight weeks old). In order to make them immunosuppressed, dexamethasone (a type of corticosteroid) was used. Indeed, causing candidiasis followed according to a specific

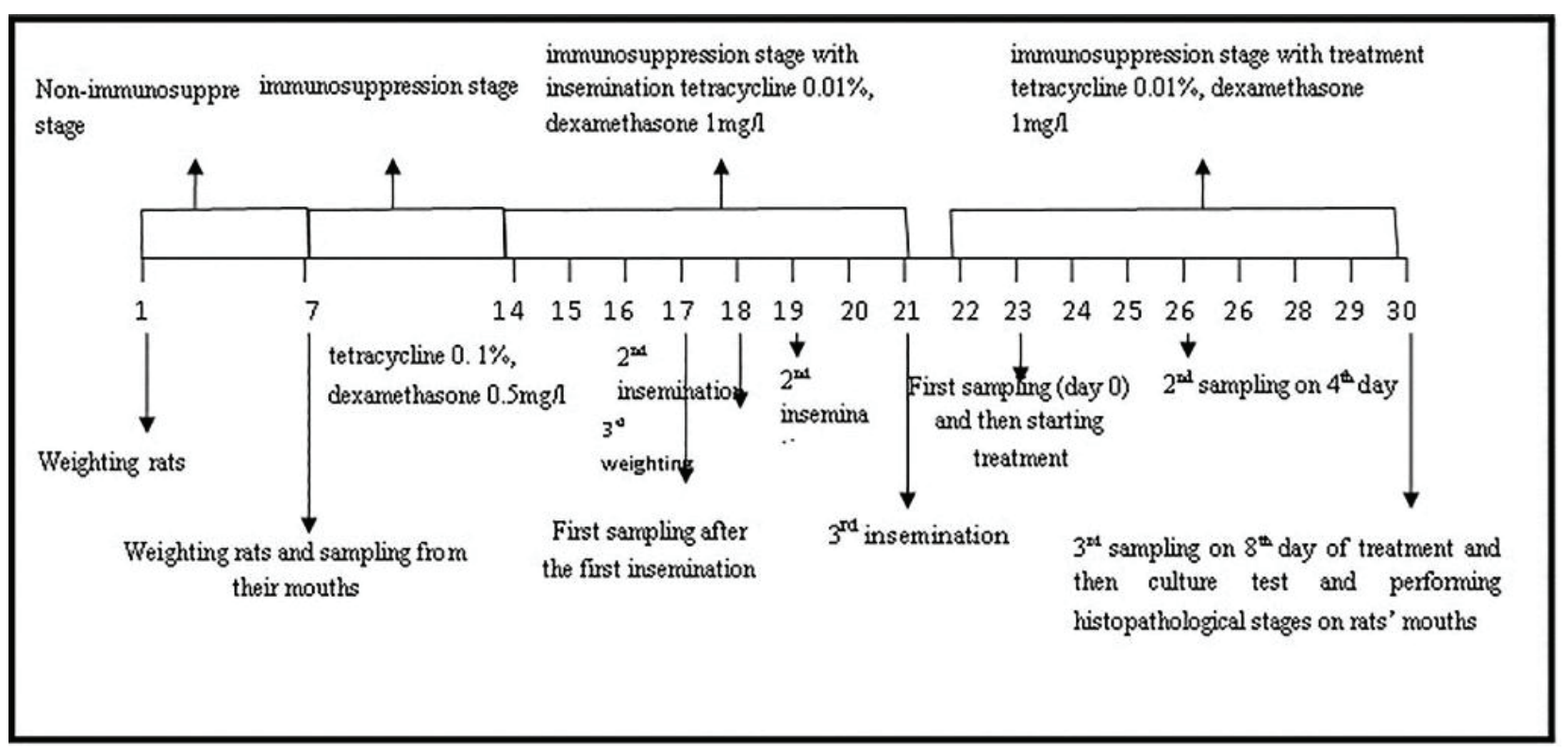

Figure 1.

The method of conducting oral candidiasis examination in rats 
timetable (fig. 1) [19-21]. In the first week, in order to adapt the animals to the new conditions and reduce the stress, they were not immunosuppressed, Water ad libitum and food plus suitable conditions were prepared for them. They were fed cool boiled water and pelted food free from fungal agents. The animals were weighted at the 1st and 7th day. The average weight was $200 \pm 20 \mathrm{~g}$ and no visible weight increase was observed. Also on the 7th day, an oral swab sample was taken from their mouth by a sterile swab so that the least insignificant candida was detected in their oral cavity (in order to quantify Colony Forming Unit (CFU) in the oral area before immunosuppression). Second week: during this week, suppression of the immune system in those rat groups that were to be immunosuppressed commenced. For this reason, $0.5 \mathrm{mg} / \mathrm{l}$ dexamethasone powder with $0.1 \%$ tetracycline (to prevent bacterial infections) were added to their drinking water. Third week: during this week, dexamethasone dose was increased to $1 \mathrm{mg} / \mathrm{l}$ and that of tetracycline was reduced to $0.01 \%$. These figures stayed constant until the end of the experiment. During the 3rd, 5th and 7th days of this week, candida suspension insemination commenced. At the end of the 3rd week, the rats were weighted again and it was witnessed that the immunosuppressed rats had an average of $65 \mathrm{~g}$ weight loss and that those rats with no immunosuppression the average weight rose to $280 \mathrm{~g}$. This is a symptom of immunosuppression. Of other symptoms of immunosuppression, severe hair fall, enlarged testicles, diarrhea, severe reduction of mobility and isolation of rats can be mentioned. In this stage, different parts of the rats' mouths were examined and they exhibited symptoms of candidiasis [21].

In this study, 35 Wistar rats (Razi Research Center) have been used according to following description: the first group included 7 rats, immunosuppressed and infected with oral candidiasis, treated with a medical formulation with SKJO twice a day for a week. The second group included 7 rats, immunosuppressed and infected with oral candidiasis and treated with a medical formulation with nystatin (positive control) twice a day for a week. The third group included 7 rats, immunosuppressed and infected with oral candidiasis and treated with a medical formulation with placebo twice a day for a week. The fourth group included 7 rats, infected with oral candidiasis but not immunosuppressed - to study the effect of immune system suppressors on the spread of infection. The fifth group included 7 rats, immunosuppressed but not infected as a control group to illustrate the role of insemination.

The level of infection was analyzed microbiologically and histopathologically. The microbiological analysis was performed in five stages: before immunosuppression, after immunosuppression and before insemination and on the 1st day (after insemination and prior to treatment) and at 4 th and 8th day of treatment with oral samples taken from rats. Then, CFU was measured. The infection level and the effectiveness of drugs were investigated by counting the number of C. albicans organisms in one milliliter of the sample (CFU) in groups treated with antifungal agents and comparing it with untreated control subjects at the end of the test. Sampling and measurement of CFU were performed according to Chamy approach [18]. The histopathological assessment was performed on the 8th day of treatment (24 $\mathrm{h}$ after last treatment day) and the animals were killed with high doses of ether. Then, their mouth and faces were disinfected using alcohol-dipped cotton and an incision was made by a scalpel from one side of their mouth to the end of their ear. Their tongue was pulled out using sterile scissors and forceps to make tissue cross-sections. After fixation and tissue processing, they were colored using hematoxilyn approach (Eosin and Periodic Acid Schiff (PAS).

The experimental protocols to perform this study were approved by the Ethics Committee for the Use of Animals of Tarbiat Modares University, School of Medical Sciences, Tehran, Iran (approval code number: 10079060; March 09, 2012). All efforts were made to minimize the discomfort of the animals and reduce the number of experimental animals. All procedures conformed to the ethical guidelines for the care and use of laboratory animals, published by the IASP and the National Institutes of Health.

\section{Statistical analysis}

In order to compare the significance of difference between placebo group and other groups, independent $\mathrm{t}$-test was used and for assessment of the difference between groups in the process of treatment 
with drugs, one-way ANOVA test was used [18]. In order to determine the reduction in each group's CFU in comparison with placebo group, first, the percentage of reduction of each group compared to the first day was calculated and then the percentage of reduction of the placebo group on 4 th and 8th days was reduced from the similar percentage of each group at the same day.

\section{RESULTS}

\section{Compounds of the essential oil}

The determination of compounds of S. khuzestanica essential oil was performed with use of GCMS approach using GC-MASS chromatograph (Hp6890 model) with column HP-5MS (30 m $\times$ $0.25 \mathrm{~mm}$ ). The type and amount of compounds in the essential oil was determined after the analyze of the chromatography (tab. 1). It was shown that carvacrol is the main a phenolic compound of this essential oil possessing high antifungal properties.
This essential oil also contains eugenol, thymol, parasmyne and $\beta$-bisabolene.

\section{Measurement of MIC and medical formulation}

In order to assess the anti-candidiasis effects of SKJO on immunosuppressed rats, first MIC and MFC of the essential oil and nystatin (positive control) were determined in vitro. Then, according to this index, the medical formulation to treat immunosuppressed rats was made (tab. 2).

According to the table, it is evidenced that the antifungal properties of nystatin are 50 times stronger than that of SKJO. In order to treat rats, a density twice the MIC of SKJO and 10 times the MIC of nystatin (as a positive control) was used.

In oral examination of three groups of rats $(n=21)$ on day 0 , the animals were severely infected with oral candidiasis and in most rats lesions in from of small, yellowish cheesy bulging plaques were visible throughout the posterior oral mucosa. The clinical manifestation of candidiasis is in form of thrush

Table 1.

The compounds in the indigenous herb Satureja khuzestanica with quantities [\%]

\begin{tabular}{llrr}
\hline Row & Compound & Retention indices & Percent \\
\hline 1 & $\alpha$-Pinene & 935 & 0.119 \\
\hline 2 & $\alpha$-Terpinene & 1019 & 0.113 \\
\hline 3 & $\mathrm{p}$-Cymene & 1026 & 0.743 \\
\hline 4 & $\sigma$-Terpinene & 1062 & 0.148 \\
\hline 5 & 4 -Terpineol & 1183 & 0.325 \\
\hline 6 & Thymol & 221 & 0.546 \\
\hline 7 & Carvacrol & 237 & 90.37 \\
\hline 8 & Eugenol & 13377 & 3.56 \\
\hline 9 & Phenol,2-methoxy-4(2-propenyl) & 1367 & 0.965 \\
\hline 10 & $\beta$-Caryophyllene & 1428 & 0.205 \\
\hline 11 & Geranyl acetate & 1458 & 0.206 \\
\hline 12 & $\beta$-Bisabolene & 282 & 0.751 \\
\hline 13 & Caryophyllene oxide & 292 & 0.245 \\
\hline 14 & 3,4,5,6,7,8, Hexahydronaphthalen-1(2H)-ono,7,methyl & 0.246 \\
\hline 15 & Podocarp-7-en-3 $\beta,-$ ol, $13 \beta$-methyl & 355 & 0.326 \\
\hline 16 & 4 Methyl-2-c3-methyl-2-butenyl-furan & 1776 & 0.289 \\
\hline $\mathbf{1 7}$ & totarol-2-penana-threnal,4b,5,6,7,8,9 & 1939 & $\mathbf{0 . 8 4 4}$ \\
\hline
\end{tabular}

Table 2.

Results of MIC and MFC in Satureja khuzestanica and nystatin

\begin{tabular}{lll}
\hline MFC & MIC & Compound \\
\hline Nystatin & $4 \mu \mathrm{g} / \mathrm{ml}$ & $8 \mu \mathrm{g} / \mathrm{ml}$ \\
\hline Satureja khuzestanica essential oil & $0.2 \mathrm{mg} / \mathrm{ml}$ & $0.3 \mathrm{mg} / \mathrm{ml}$ \\
\hline
\end{tabular}


with rare red lesions in between these thrushes in some rats with napless and inflammatory mucosa in these parts similar to atrophic candidiasis (erythematous). However, this form of disease was less prevalent than thrush. These lesions completely disappeared in both treatment groups (SKJO and nystatin) after 8 days of treatment but in untreated animals and infectious groups (placebo group and group 3), these lesions remained on the tongue during the course of experiment. Also in group 4 (infected, not immunosuppressed) and 5 (immunosuppressed but not infected), no clinical symptoms were visible.

\section{Microbiological assessment}

Before starting the tests, samples were taken from the mouths of all the rats and no evidence could be found of any infection in the animals. The result of culture test for all the oral samples were negative both from the start of the test and in the immunosuppression stage before insemination with no fungal growth. The results of oral culture of the rats in group 5 i.e. the immunosuppressed yet uninfected rats (without insemination), were negative throughout the study which is indicative of the fact that in order for candidiasis to be inflicted, insemination of the candida is essential and immunosuppressed alone would not suffice. On day 0 , samples were taken from the mouths of all infected animals with significantly positive culture results (except that of non-immunosuppressed group) and the quantity of mean logarithm of CFU for each swab for all three groups (the groups treated with SKJO, the group treated with nystatin and the placebo group) stood at $4.73 \pm 0.06$ and all rats in these groups were severely infected. The one-way ANOVA showed that none of these four groups had a significant difference of infection on day 0 . Only in the immunosuppressedbut-uninfected group, 4 rats had minor infections and according to the aforementioned statistical test, these groups had a significant difference of infection as compared with placebo group $(p<0.05)$ (tab. 3 , fig. $2,3)$. On the 4 th day of treatment, samples were taken from the oral cavities of all animals, cultures were made and the quantity of mean logarithm of CFU was determined for all. All the animals treated with SKJO were still infected but showed severely reduced quantity of mean logarithm of CFU $(p<0.001)$. In the group treated with nystatin, only $5 / 7$ of the animals were infected with positive culture results with significantly reduced quantity of mean logarithm of CFU $(p<0.05)$. Also, in the groups treated with SKJO and nystatin, the quantities of mean logarithm of CFU were $85.62 \%$ and $90.62 \%$, respectively, in comparison with placebo group. As for the rats in the non-immunosuppressed group, only $2 / 7$ of animals exhibited minor infections and their quantity of mean logarithm of CFU reduced significantly $(p<0.001)$ (tab. 3, fig. 2, 3). Finally, comparison of the groups treated with SKJO and nystatin on the 4th day of treatment with one-way ANOVA illustrated that treatment with nystatin results in a significant reduction in the quantity of mean logarithm of CFU in comparison with SKJO group $(p<0.05)$ (tab. 3, fig. 2,3). On the 8th day of treatment, only $2 / 7$ of animals in the groups treated with SKJO showed minor infections in culture. Also, 1/7 of the animals in nystatin treatment group an 1/7 of those

Table 3.

Results of MIC and MFC in Satureja khuzestanica and nystatin

\begin{tabular}{|c|c|c|c|c|c|c|c|c|}
\hline \multirow{4}{*}{ Group } & \multicolumn{2}{|c|}{ Day 0} & \multicolumn{3}{|c|}{ Day 4} & \multicolumn{3}{|c|}{ Day 8} \\
\hline & Animals & Logarithm & Animals & Logarithm & Reduction & Animals & Logarithm & Reduction \\
\hline & infected & $\mathrm{CFU}$ & infected & CFU & {$[\%]$} & infected & $\mathrm{CFU}$ & [\%] \\
\hline & {$[\%]$} & $\pm \mathrm{SD}$ & {$[\%]$} & $\pm \mathrm{SD}$ & $\mathrm{CFU}$ & [\%] & $\pm \mathrm{SD}$ & $\mathrm{CFU}$ \\
\hline Placebo & $7.7(100)$ & $4.73 \pm 0.08$ & $7.7(100)$ & $4.71 \pm 0.06$ & - & $7.7(100)$ & $4.69 \pm 0.06$ & - \\
\hline S. khuzestanica & $7.7(100)$ & $4.75 \pm 0.06$ & $7.7(100)$ & $3.74 \pm 0.04$ & 85.62 & $2.7(28.57)$ & $2.58 \pm 0.07^{\star *}$ & 91.51 \\
\hline Nystatin & $7.7(100)$ & $4.71 \pm 0.04$ & $5.7(71.43)$ & $3.74 \pm 0.04^{*}$ & 90.65 & $1.7(14.28)$ & $2.5^{\star *}$ & 91.51 \\
\hline Non- & & & & & & & & \\
\hline immunosuppressed & $4.7(57.1)$ & $3.12 \pm 0.3^{*}$ & $2.7(28.57)$ & $2.76 \pm 0.16^{\star *}$ & 68.71 & $1.7(14.28)$ & $2.26^{* *}$ & 84.09 \\
\hline and infected group & & & & & & & & \\
\hline
\end{tabular}




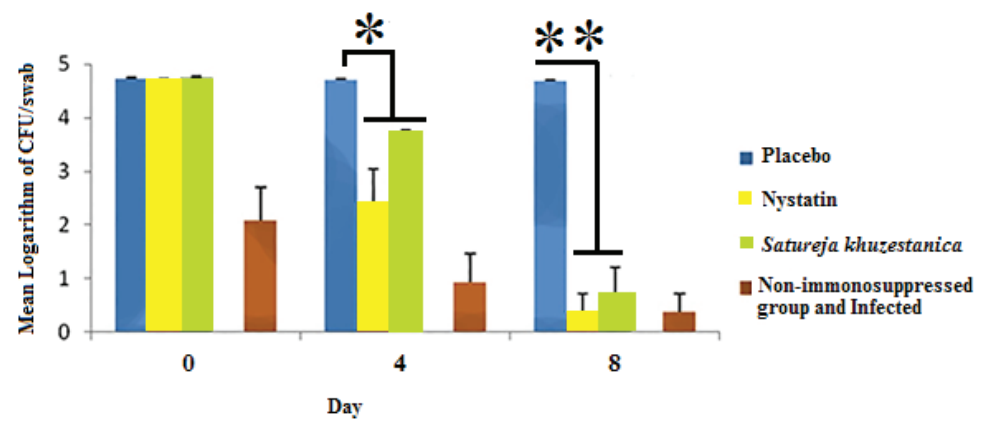

* - difference with the placebo group at the significance level of $p<0.05$

** - difference with the placebo group at the significance level of $p<0.001$

Figure 2.

Comparison of the effectiveness of different treatment groups since the start of treatment until the 8th day (in columns)

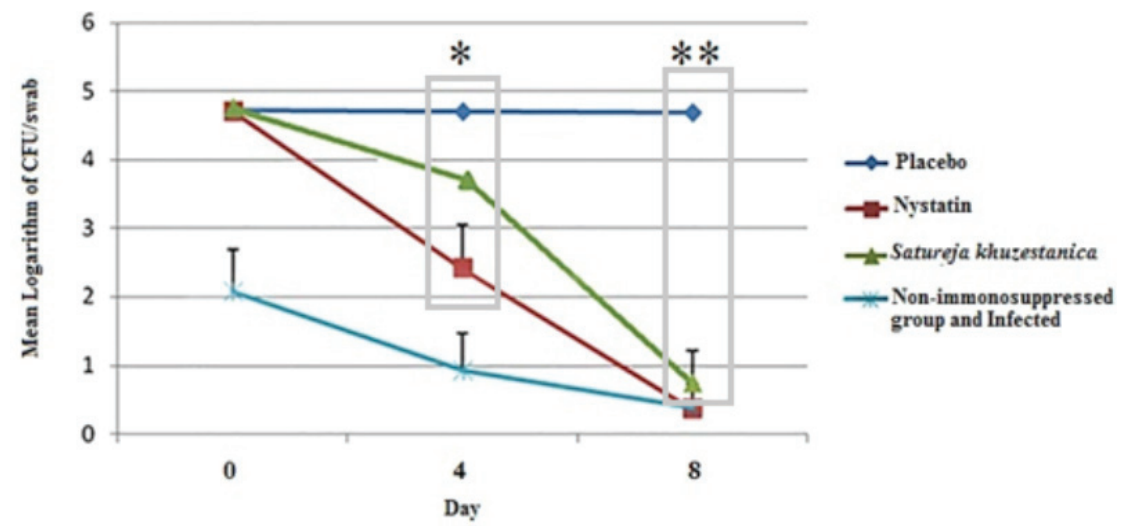

* - difference with the placebo group at the significance level of $p<0.05$

** - difference with the placebo group at the significance level of $p<0.001$

Figure 3.

Comparison of the effectiveness of different treatment groups since the start of treatment until the 8th day (linear)

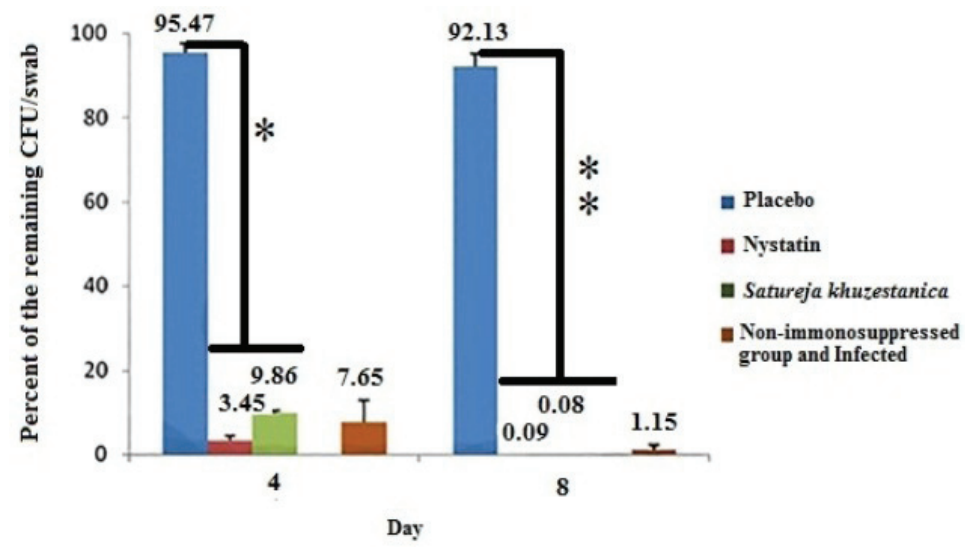

* - difference with the placebo group at the significance level of $p<0.05$

** - difference with the placebo group at the significance level of $p<0.001$

Figure 4 .

Comparison of the percentage of decrease in the infection of tested groups on 4 th and 8 th days with day 0 
in the non-immunosuppressed group had minimally positive cultures. In addition, in comparison with the placebo group, a significant change was observed in the quantity of mean logarithm of CFU of the groups treated with SKJO $(91.51 \%)(\mathrm{p}<0.001)$ (tab. 3, fig. 3). Treatment with nystatin was particularly effective in eliminating candida organisms as in the 8th day. Only $1 / 7$ of animal cultures were positive and their quantity of mean logarithm of CFU reduced significantly $(\mathrm{p}<0.001)$. Comparison of the groups treated with SKJO and nystatin on the 8th day of treatment with one-way ANOVA illustrated that there is no significant difference between them (tab. 3, fig. 4).

\section{Histopathological assessment}

After studying the histopathological lams acquired from the back of tongues of the 3 treatment groups, the following were found: in the negative control animals with untreated infections (group 3, placebo group), vast colonizations of fungal cells and hyphae were visible on the surface of dorsal tongue epithelium and corneum. The hyphae had penetrated into epithelium and even the deeper layers with fluctuating thickness. The histopathological properties suggested infection in this group.

In groups of animals treated with SKJO and nystatin (groups 1 and 2), no fungal elements were observed in form of yeast or hyphae on the dorsal
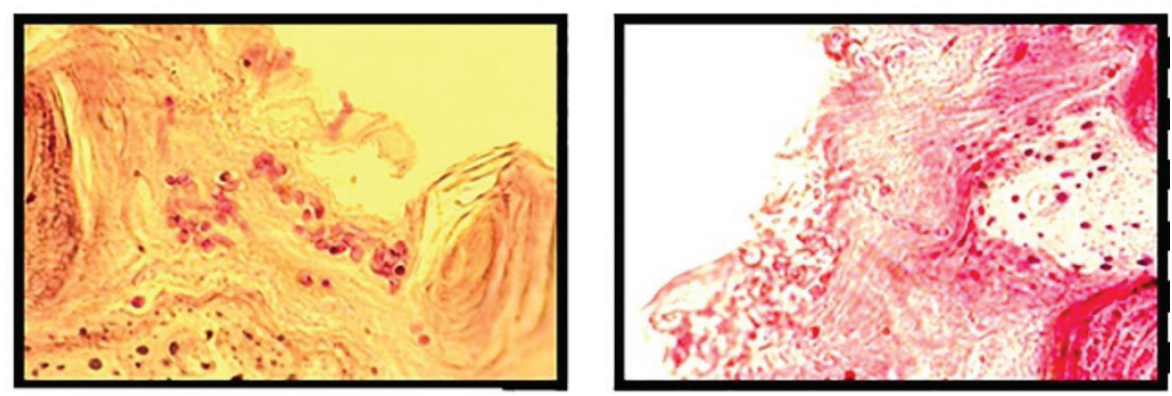

Figure 5.

Tongue histopathology of rats in the placebo group (3) infected with oral candidiasis with PAS coloring (right) and H\&E coloring (left) magnified with No. 40 lens
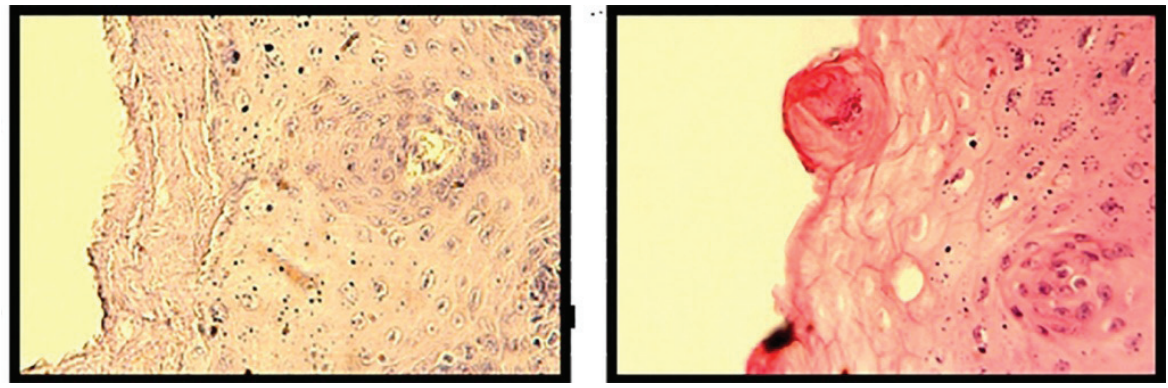

Figure 6.

Tongue histopathology of rats in the groups treated with Satureja khuzestanica (1) infected with oral candidiasis with PAS coloring magnified with No. 40 lens (right) and H\&E coloring (left) with x100 magnification
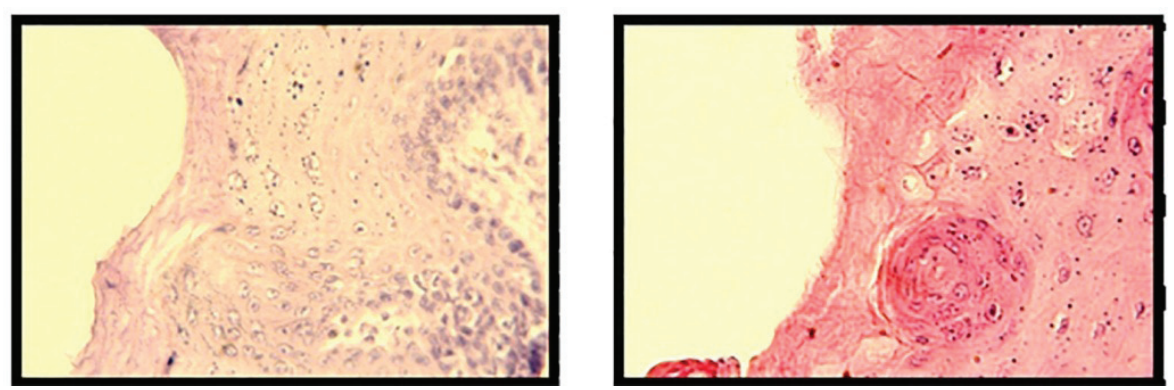

Figure 7.

Tongue histopathology of rats in the groups treated with nystatin (2) infected with oral candidiasis with PAS coloring magnified with No. 40 lens (right) and H\&E coloring (left) with $100 \mathrm{x}$ magnification 
tongue epithelium. Also, histopathological properties suggested no infection in these groups who had clean and normal dorsal tongue mucosa (fig. 5-7).

\section{DISCUSSION}

At the beginning stages of this study, the compounds in S. khuzestanica produced via steam distillation with Clevenger apparatus, were identified using GC-Mass approach. The main compound of this herb is a phenolic compound possessing high antifungal properties called carvacrol. This compound forms more than $90 \%$ of this essential oil's content and it also contains eugenol, thymol, parasmyn, and $\beta$-bisabolene. These findings were in agreement with other studies [15, 22, 23].

Carvacrol reacts with the cell membrane through changing penetrability of channels $\mathrm{H}^{+} / \mathrm{K}^{+}$and leads to the secretion of ATP. Alternations in ionic gradient will result in halting and disruption of cell functions and ultimately its death. Thymol is a carvacrol isomer [24]. This element prevents the activity and survival of fungal hyphae and affects the candida's membrane and metabolism, probably affecting the fungal cell membrane [25]. In other research, it has been suggested that carvacrol prohibits the germination and formation of C. albicans hyphae [26]. Antifungal effects of eugenol have been approved in numerous studies. Parasmyn is a precursor for thymol and carvacrol with no antimicrobial effects [27]. In order to make a medical formulation for treatment of candidiasis in immunosuppressed rats in present study, first the MIC of SKJO and nystatin (positive control) was calculated using in vitro micro-dilution. The MIC value for SKJO for this clinical pathogen isolate was $0.2 \mathrm{mg} / \mathrm{ml}$. Also, the MIC of nystatin for stopping the growth of this clinical pathogen isolate was $4 \mu \mathrm{g} / \mathrm{ml}$. In other words, the antifungal effect of nystatin is 50 times higher than that of SKJO. These findings are in partial agreement with those of other studies except that of our study: a clinical pathogen isolate was used, whereas other studies used standardized isolates [22]. Also, the MFC value for SKJO was $0.3 \mathrm{mg} / \mathrm{ml}$ and that of nystatin stood at $8 \mu \mathrm{g} / \mathrm{ml}$. In order to achieve a medicinal formulation for SKJO, final density twice the MIC of SKJO and for nystatin, 10 times the MIC of nystatin was used to compare the treatment effects of the A standard experimental model was used in this study according to Martinez model to assess the antifungal effects of SKJO and nystatin (positive control) on oral candidiasis in immunosuppressed rats which is easy to produce [18-20]. Systemic prescription of dexamethasone in drinking water two weeks before treatment led to a severe reduction of leukocytes in bloodstream and neutropenia in rats. This drug acts by preventing the accumulation of swollen cells in the inflammatory area, inhibition of phagocytosis and release of chemical mediators of inflammation, leading to severe suppression of immune system in the rats. Moreover, prescription of systemic antibiotics like tetracycline will facilitate the development of oral candidiasis in rats and prevents bacterial infections $[28,29]$. Some of the symptoms of immunosuppression in rats are severe weight loss, hair loss, enlargement of testicles, diarrhea, reduced mobility and isolation [21].

In this study, the researchers have used a highly potent $C$. albicans isolate to induce oral candidiasis acquired from a patient suffering from acute vaginal candidiasis. The results of measuring CFU and clinical examinations on day 0 of treatment and also high level of infection in untreated (placebo) rats throughout the study were indicative of its high potency. This isolate possessed the ability for tube germ and hyphae formation that were proven by the results of histopathology. Tube germ formation visible earlier than hyphae in candidiasis is in general related to the ability to attach to epithelial cells [30] and resistance to phagocytosis due to their large size. It was suggested in many studies that hyphae structures act better than germinating cells in acquiring a fixed position in the process of preliminary host intrusion [31]. These views have led to the idea that formation of a generative and hyphae tube in C. albicans will lead to deeper penetration in epithelium and is significant in causing the disease [32]. This model is also useful in studies evaluating the antifungal effects in treatment of vaginal candidiasis and is also productive in the treatment of systemic candidiasis with only minor alterations [18-21].

It can be shown from the results of 8 days of microbiological and histopathological assessment that although in some studies it was mentioned that rats can shelter C. albicans on a lower level as transient commensalism and that any researcher who uses this species must also consider abnormal oral colonization of candida before insemination [33,34]. The results of oral culture of all the rats in this study were negative both at the start and in the immunosuppressed stage, before the insemination. This result is in agreement with that of many researchers that used rats for oral candidiasis [18-20].

Since in group 4 (non-immunosuppressed but infected and inseminated) only $1 / 7$ of animals 
showed minimally positive cultures and also since whole group 5 (immunosuppressed but uninfected and not inseminated) had negative cultures with no signs of oral candidiasis in these two groups, it can be concluded that in order for a successful oral candidiasis model to be formulated for rats, immunosuppression and insemination should be performed simultaneously and both of them are necessary stages. In three treatment groups (1,2 and 3) the CFU logarithm was calculated on day 0 from oral culture samples of all rats $(n=28)$ which was similar to the results of other studies with use of the same inseminator [17] and all rats were infected and approved clinical symptoms of oral candidiasis [1820]. In untreated infected immunosuppressed group (group 3 or placebo), the culture samples of all animals were positive during the 8-day course of study and the rats exhibited a steady infection all time (with $8 \%$ reduction at the last day in comparison to day 0 ). The histopathologic observations also indicated abundant hyphae and germinating elements and the penetration of hyphae elements into the epithelium of dorsal tongue area that was in agreement with microbiological findings. Also clinical lesions of oral candidiasis in all rats of this group during the course of treatment in 8 days indicated a steady infection in them. Four days after treatment with $S$. khuzestanica essential oil, the animals were still infected but their CFU logarithm value showed $85.62 \%$ reduction in comparison to the placebo group. On the other hand, 4 days after treatment with nystatin, only the culture of 5/7 of cases came out minimally positive with a $90.62 \%$ reduction in their CFU compared with that of placebo group that is indicative of more significant effect of nystatin during first 4 days of treatment. On the 8th day, only $2 / 7$ of animals treated with SKJO had minimally positive cultures with no clear signs of oral candidiasis in dorsal area of their tongues and no germinating or hyphae elements in their histopathologic assessments. The microbiological results were approved by the histopathologic ones and the treatment of infection was $100 \%$ successful. Nystatin treatment was significantly successful and only $1 / 7$ of animals showed minimal positive cultures on the 8th day of treatment. The microbiological results were approved by the histopathologic ones and the treatment of infection was successful in this group as well.

\section{CONCLUSIONS}

It can be concluded that treatment of oral candidiasis by with S. khuzestanica essential oil in 2-fold MIC density was successful and approved by microbiological and histopathologic assessments and clinical examinations, equal to 8 days of treatment with nystatin at 10 -fold MIC density. This probably can be justified by the fact that main compound of SKJO is carvacrol (higher than 90\%) that is a phenolic compound with strong antifungal properties. With quick evaporation of this material in the oral cavity and its high penetrability, the cells of this compound can penetrate remote areas of the tongue (like grooves and villi and deep parts) and with a density twice the value of MIC, this essential oil can have an effect equal to 10-fold MIC of nystatin and remove the clinical symptoms of oral candidiasis on rats' tongues [18]. The dosage of SKJO in this study is far less than its lethal dosage $\left(\mathrm{LD}_{50}\right)$. The lethal dosage of $S$. khuzestanica essential oil in rats is higher than $2 \mathrm{mg} / \mathrm{kg}$ [35].

\section{ACKNOWLEDGEMENT}

We would like to thank Khorraman Pharmaceutical Co. and Tarbiat Modares University, School of Medical Sciences, Tehran, Iran for financial support. We specially thank Dr. Alinazar Salehnia and Dr. Alireza Pesarakloo for their unforgettable help in this study. Animals were kindly provided by the Animal Maintenance Unit of Razi Research Center, Karaj, Iran.

Conflict of interest: Authors declare no conflict of interest

\section{REFERENCES}

1. Wingard JR. Importance of candida species other than C. albicans as pathogens in oncology patients. Clin Infect Dis Jan 1995; 20 (1):115-25. doi: http://dx.doi.org/10.1093/clinids/20.1.115

2. Calderon RA, Fonzi WA. Virulence factor of Candida albicans. Trends Microbiol 2001; 327-335. doi: http:// dx.doi.org/10.1016/S0966-842X(01)02094-7

3. Singh N. Trends in the epidemiology of opportunistic fungal infections: predisposing factors of antimicrobial use practices (Review article). Clin Infect Dis 2001; 33:1692-1696. doi: http:// dx.doi.org/10.1086/323895 
4. Samaranayake LP. Oral mycoses in HIV infection. Oral Med Oral Pathol 1992; 73: 171180. doi: http:// dx.doi.org/10.1016/00304220(92)90191-R

5. Dignani MC, Solomkin JS, Anaissie EJ, Candida. In: Anaissie EJ, McGinnis MR, Pfaller MA. Clinical Mycology. Churchill Livingstone 2003:195-239.

6. Samaranayake LP, Lamey PJ. Oral candidosis: 1 . Clinicopathological aspects. Dent Update 1988; 15:227-228, 230-231.

7. Mac Phail LM, Green Span D, Dodd CL, Heinic GS, Beck C, Ekoku E. Association of fungal species with oral candidiasis in HIV infection. J Dent Res 1993; 72:353.

8. Samaranayake YH, Samaranayake LP. Experimental oral candidiasis in animal models. J Clin Microbiol 2001; 14: 398-429. doi: http://dx.doi. org/10.1128/CMR.14.2.398-429.2001

9. Ellepolla A N B, Samaranayake LP. Oral candidal infections and antimycoties. Crit Rev Oral Biol Med 2000; 11:172-198.

10. Kauffman CA, Carver PL. Antifungal agents in the 1990s. Current status and future developments. Drugs 1997; 53:539-549. doi: http:// dx.doi.org/10.2165/00003495-19975304000001

11. Ahmad S, Khan Z, Mustafa AS, Khan ZU. Seminested PCR for diagnosis of candidemia: comparison with culture, antigen detection, and biochemical methods for species identification. J Clin Microbiol 2002; 40(7):2483-2489. doi: http://dx.doi.org/10.1128/JCM.40.7.24832489.2002

12. Ghasemi Dehkordi N. Iranian Herbal Pharmacopoeia (IHP). Min Health Pub 2002; 2:749-753.

13. Abdollahi M, Salehnia A, Mortazavi SH, Ebrahimi M, Shafiee A, Fouladian F, et al. Antioxidant, antidiabetic, antihyperlipidemic, reproduction stimulatory properties and safety of essential oil of Satureja khuzestanica in rat in vivo: a toxicopharmacological study. Med Sci Monit 2003; 9: BR331-335.

14. Amanlou M, Fazeli MR, Arvin A, Amin HG,
Farsam H. Antimicrobial activity of crude methanolic extract of Satureja khuzestanica. Fitoterapia $2004 ; 75: 768-770$.

15. Radpour M. Identification and structure of native and cultivated Satureja khuzestanica using GC-MS. Professional Doctorate Thesis, Faculty of Pharmacy, Teheran University of Medical Sciences, Thesis No. 4261, 2002.

16. National committee for clinical laboratories standard. Reference method for broth dilution antifungal susceptibility testing of yeasts. Approved standard. National committee clinical laboratories standard, 1997.

17. Evans EGV, Richardson MD. Medical mycology. A practical approach. Oxford 1989.

18. Chami N, Chami F, Bennis S, Trouillas J, Remmal A. Antifungal treatment with carvacrol and eugenol of oral candidiasis in immunosuppressed rats. Braz Infect Dis 2004; 8:217-26. doi: http://dx.doi.org/ S1413-86702004000300005

19. Martinez A, Ferrer S, Santos I, Jimenez E, Sparrowe J, Regadera J, et al. Antifungal activities of two new Azasordarins, GW471552 and GW471558, in experimental models of oral and vulvovaginal candidiasis in immunosuppressed rats. Antimicrob Agents Chemother 2001; 45:3304-3309. doi: http://dx.doi.org/10.1128/ AAC.45.12.3304-3309.2001

20. Martinez A, Regardera J, Jimenez E, Santos I, Gargallo-Viola D. Antifungal efficacy of GM237354, a sordarin derivative, in experimental oral candidiasis in immunosuppressed rats. Antimicrob Agents Chemother 2001; 45:1008-1013. doi: http://dx.doi.org/10.1128/ AAC.45.4.1008-1013.2001

21. Üner A, Nceboz T, Uysalci M, Dagci H. Immune deficiency and cryptosporidiosis in rats. Turk J Vet Anim Sci 2003; 27:1187-1191.

22. Sepahvand A. Effects of antifungal Satureja Khuzestanika using in vitro in Lorestan province. MS, Faculty of Health and Institute for Health Research, Tehran University of Medical Sciences, Thesis No. 5348, 2004.

23. Ultee A, Kets EPW, Smid ET. Mechanism of action of carvacrol on the food borne pathogen 
Bacillus cereus. Appl Environ Microbiol 1999; 65:4606-4610.

24. Amin GH, Salehi MH, Zahedi M, Khanavi M, Samadi N. Essential oil composition and antimicrobial activity of Oliveria decumbens. Fitoterapia 2005; 76:704-707.

25. Braga PC, Alfieri M, Culici M, Dal Sasso M. Inhibitory activity of thymol against the formation and viability of Candida albicans hyphae. Mycoses 2007; 50:502-506. doi: http:// dx.doi. org/10.1111/j.1439-0507.2007.01412.x

26. Manohar V, Ingram C, Gray J, Tlpur NA, Echarad BW, Bagchi D et al. Antifungal activities of origanum oil against Candida albicans. Mol Cell Biochem 2001; 228:111-117. doi: http://dx.doi. org/10.1023/A:1013311632207

27. Cosentino S, Tuberose CIG, Pishao B, Satta M, Mascia V, Arzedi E, Palmas F. In vitro antimicrobial activity and chemical composition of Thymus sardinian essential oils. Lett Appl Microbiol 1999; 29:130-135. doi: http://dx.doi. org/10.1046/j.1472-765X.1999.00605.x

28. Grainger W, Presteril E, Schneeweiss B, Teleky B, Georgopoulos A. Treatment of Candida albicans fungamia with fluconazole. J Infect 1993; 26:133-146.
29. Russell C, Jones J. Effects of oral inoculation of Candida albicans in tetracycline-treated rats. J Med Microbiol 1973; 6:275-279.

30. Kimura LH, Pearsall NN. Relationship between germination of Candida albicans and increased adherence to buccal epithelial cells. Infect Immun $1980 ; 28: 464-468$.

31. Martinez MV, Craig GT, Lamb DJ. An investigation of the role of true hypha production in the pathogenesis of experimental oral candidiosis. Sabouraudia 1984; 22:471-476.

32. Odds FC. Candida and candidiasis. Leicester 1979.

33. Winner HI, Hurley R. Candida albicans. London 1964.

34. O'Grady JF, Reade PC. Role of thermal trauma in experimental oral mucosal candida infection in rat. J Oral Pathol Med 1993; 22: 132-137. doi: http://dx.doi.org/10.1111/j.1600-0714.1993. tb01044.x

35. Arvin A. Phytochemistry, typography and study of effects of antimicrobial and antifungal Satureja khuzestanika. Indigenous and cultivated. Professional Doctorate Thesis, Faculty of Pharmacy, Tehran University of Medical Sciences, Thesis No. 4360, 2002-82. 